\title{
Signal Transmission and Representation Based on High Performance Event Timing
}

\author{
Ivars BILINSKIS, Eugene BOOLE \\ Institute of Electronics and Computer Science \\ 14 Dzerbenes St., LV-1006, Riga, Latvia. \\ bilinskiseedi.lv, bulsdedi.lv
}

\begin{abstract}
Specifics and advantages of using high performance event timing for signal transmission and representation in the time and frequency domains are described. The focus is on the methods and systems for energy-efficient computing of Discrete Fourier Transform based on replacing the sinusoidal basis functions by binary-valued rectangular functions. The sources of potential errors are revealed and methods for avoiding them are discussed and evaluated.
\end{abstract}

Keywords: event timing, Fourier coefficient estimation, digital signal processing

\section{Introduction}

Using timing information for signal digitizing and representing in the time and frequency domains and measuring various parameters of them has been known for a long time. The first developed and more popular is the approach based on timing the time instants of signal zero-crossing events. It is discussed, in particular, in (Curtis and Oppenheim, 1987; Kumaresan and Wang, 2001) where many references to publications in this area are given. Using the timing information obtained at timing signal crossings of reference functions, including sine-waves, are also considered and discussed in (Bilinskis and Sudars, 2008a, 2008b) as it is also done within the framework of this paper.

Actually signal crossings of well-chosen and precisely generated reference functions might carry much more information than timing the zero-crossings during the same time interval. Various types of waveforms can be used for getting this type of information. At first glance application of saw-tooth waveforms for that theoretically is most appropriate. However experience gained at using the event timing shows that in practise it is much better to use the sine-wave reference functions as they could be precisely generated electronically at much higher frequencies in a considerably simpler way. Various aspects of signal digitizing based on timing the signal and sine-wave crossing (SWC) events have been studied and are described in (Bilinskis, 2007).

Considerably less attention, in general, has been paid to the problem of achieving high precision at the crossing event timing by using specific high performance Event Timers capable of performing picosecond resolution event timing at sufficiently high repetition rate of these events. In our earlier publications (Bilinskis et al., 2013a, 2013b) 
we have drawn attention to using Event Timer A033-ET as an effective tool for applications in the field of signal digitizing and transmission over optical lines.

Maturity and competitiveness of this Event Timer have been proven by successful using it for Satellite Laser Ranging in many countries worldwide in the framework of the International Laser Ranging Service network. Experience accumulated over last 20 years of using the Event Timer for Laser Ranging in about 30 Satellite Laser Ranging stations around the world confirms the high application potential for building and exploiting various systems with this Event Timer in the core (Zhongping et al., 2008; Artyukh et al., 2011).

Conditions for signal transmission and representation in time and frequency domains can be flexibly varied by choosing and using an appropriate method for signal digitizing based on event timing. That is explained in (Sudars et al., 2015). Various aspects of using picosecond resolution event timing at data acquisition and transmission over optical lines are described and discussed in (Bilinskis et al., 2015; Sudars et al., 2016; Bilinskis et al., 2016).

This paper addresses the topic of representing signals encoded by Pulse Width Modulation, transmitted and then reconstructed by using high performance event timers. Specifics of such signal transmission and representing them in the Time and Frequency domains are considered in Section 2 and Section 3, respectively. The topic of this paper is related to the work discussed in (Bilinskis et al., 2015; Sudars et al., 2016; Bilinskis et al., 2016) and it is focused on research and development of innovative approaches to Fourier coefficient estimating with significantly improved precision and applicability in a widened frequency range. Specifically, application of input signal heterodyning is described in Section 4 and the proposed method for reducing noise at DFT by using different rates at sampling the input signals and the rectangular basis functions is discussed in Section 5. The obtained results are summarised in Section 6.

\section{Specifics of transmitting the timing information}

Specific conditions for representing the transmitted signals in the time and frequency domains obviously depend on the used approaches to encoding and transmitting the timing information. In the considered case that is done on the basis of using high performance event timers.

\subsection{Two approaches to transmitting the timing information}

The structure of the whole system used for information transmission, in this case, is given in Fig. 1. Two types of converters are used, specifically, the Analog-to-Event Converter (AEC) and Event-to-Digital Converter (EDC), as it is shown in Fig. 1a. Picosecond resolution event timers are in the core of both of these converters and the event timing function plays a very important role at signal encoding (performed by AEC) and at reconstruction the transmitted signal carried out by the Receiver. The block diagram of the Receiver is given in Fig. 1b.

What are the features of the transmitted signal first of all depends on the approach to signal encoding used on the transmitter side for supporting precise forming of the pulses carrying the information of the signal and sine-wave reference crossing (SWC) events. Transmitting the information obtained in result of direct timing the SWC events then is performed by using the Pulse Position Modulation (PPM). Although this approach has 
significant advantages and provides for obtaining better results than the widely used zero or level crossing approaches, it has also a significant drawback. Indeed, the transmitted pulses then carry the information of both the signal sampling time instances and the respective sample values as it is described, for example, in (Bilinskis et al., 2013a). That actually complicates digital processing of the transmitted information as the SWC event streams are non-uniform.

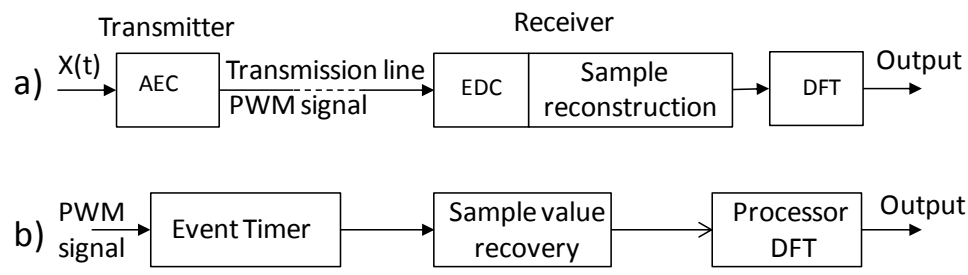

Fig. 1. Block diagrams illustrating the system used for transmitting event timing information: (a) structural diagram of the transmission system; (b) diagram of the receiver structure.

In this paper we consider the approach to transmitting the timing information described in (Sudars et al., 2015), which does not have the mentioned drawback. According to this approach, a Track\&Hold device is used for periodic sampling of the input signal and the events that are timed are formed as the Track\&Hold output level crossings with the sine-wave reference function. Then the timing information related to signal sample values are taken periodically at predetermined time instants $t_{k},(k=0,1,2$, ...) and the mentioned crossing events carry only the information of these sample values $x\left(t_{k}\right)=x_{k}$. In this case transmitting the timing information is performed by using the Pulse Width Modulation (PWM) as it is shown in the diagrams given in Fig.1.

\subsection{Reconstruction of the transmitted signal}

According to the mentioned second approach, the transmitted signal is represented in the Time domain by the input signal sample values $x_{k}$. They have to be recovered from the transmitted width modulated pulse stream. Demodulation of the received PWM signal is performed by the Event-to-Digital Converter (EDC) added to the Receiver. EDC, used for demodulation, in this case performs timing of the falling edges of the transmitted and received pulses, providing for recovery of signal sample value as 12 bit numbers.

Compressive information transmission is achieved as each transmitted single pulse carries a 12 bit word and that makes it possible to transmit event timing information at $300 \mathrm{Mbps}$ bit rate (Bilinskis et al., 2015). The quantization step of the transmitted pulses then is equal to $6.1 \mathrm{ps}$. To achieve information compression at transmission over the optical transmission lines on that level, using the picosecond resolution high-speed Event Timer A033-ET systems is essential. It supports time interval measurements between two events with the typical RMS errors in the range of 3.5 to 4 ps with errors of a single time measurements approximately in the range of 2.3-2.8 ps. Capabilities of this Event Timer are described, in particular, in (Artyukh et al., 2011). Design and performance of Event Timer A033-ET are on high level and that has been proved by successful meeting the demands dictated by practical applications of these systems in many countries worldwide in the framework of the International Laser Ranging Service network.

Let us consider in some detail the process of transforming the received PWM signals into the representative digital signals under the specific conditions related to periodic 
sampling of the input signals. The sampling time instants $t_{k},(k=0,1,2, \ldots)$ mark the leading edges of PWM pulses. They are fixed as the transmitter and receiver time scales are synchronized. Using synchronized clocks on both sides provide for adequate measurements of the pulse widths $w_{0}, w_{1}, \ldots, w_{7}$ proportional to the respective sample $x_{0}, x_{1}, \ldots, x_{7}$ of the transmitted analog signal. Only the trailing edges of these pulses then have to be timed at demodulation of the PWM pulse train performed in the process of the signal sample value $x_{k}$ recovery. The Event Timing block, shown in Fig.1b, performs precise and fast event timing function needed for demodulation. It means that it measures the time instants $\tau_{k}$ when the signal sample value level $x_{k}$ (output signal of the $\mathrm{T} / \mathrm{H}$ device) and the reference function crossing events occur. Under these conditions the signal sample value $x_{k}$, representing the transmitted in the Time domain, are calculated as

$$
x_{k}=A_{r} \cos \left[2 \pi f_{r}\left(\tau_{k}-t_{k}\right)\right]
$$

where $A_{r}, f_{r}$ are the amplitude and frequency of the reference sine-wave.

\section{Representing the transmitted signals in the frequency domain}

Representing the transmitted signals in the frequency domain, when it is based on application of the high performance event timers, evidently is specific. It has been discussed before, for example, in papers (Sudars et al., 2016; Bilinskis et al., 2016). This paper is focused on development of computationally and energy efficient approach to exploiting Discrete Fourier Transform (DFT) for obtaining representative signal parameter estimates.

\subsection{Considered options}

The classical approach to estimating the Fourier coefficients $a_{i}, b_{i}$, characterizing digital signals $x\left(t_{k}\right)=x_{k}, k=0,1,2, \ldots, N$ in the frequency domain at frequencies $f_{i}, i=0,1,2, \ldots, M$ is based on calculations performed according to the following equations:

$$
\left\{\begin{array}{l}
a_{i}=\frac{2}{N} \sum_{k=0}^{N-1} x_{k} \cos \omega_{i} t_{k} \\
b_{i}=\frac{2}{N} \sum_{k=0}^{N-1} x_{k} \sin \omega_{i} t_{k}
\end{array}\right.
$$

where $t_{k}$ are the sampling instants and $\omega_{i}=2 \pi f_{i}, k=0,1,2, \ldots, N$.

This paper is focused on considering another option of performing DFT. It is based on replacement of $\cos \omega_{i} t_{k}, \sin \omega_{i} t_{k}$ by the functions $R_{s}\left(i, t_{k}\right)=\operatorname{sgn}\left(\sin \omega_{i} t_{k}\right)$, $R_{c}\left(i, t_{k}\right)=\operatorname{sgn}\left(\cos \omega_{i} t_{k}\right)$ and on estimating the coefficients $a_{i}, b_{i}$ as follows: 


$$
\left\{\begin{array}{l}
a_{i}=\frac{\pi}{2 N} \sum_{k=0}^{N-1} x_{k} \mathrm{R}_{\mathrm{c}}\left(i, t_{k}\right) \\
b_{i}=\frac{\pi}{2 N} \sum_{k=0}^{N-1} x_{k} \mathrm{R}_{\mathrm{s}}\left(i, t_{k}\right)
\end{array}\right.
$$

Conditions for processing signals at performing DFT according to (3) then are quite different in both the positive and negative senses. While estimating the coefficients $a_{i}, b_{i}$ in this case evidently can be done in a significantly simplified way as $R_{c}\left(i, t_{k}\right), R_{s}\left(i, t_{k}\right)$ have only the values $-1,+1$, the estimation precision is worse.

Explanation of this precision worsening fact seems to be simple and obvious if the involved rectangular functions are considered as rough approximation of the sinusoidal functions. Indeed, the errors of such approximation are large and, unfortunately, they cannot be reduced. Actually this assumption is wrong. Investigations described in (Sudars et al., 2016; Bilinskis et al., 2016) show that these approximation errors do not represent the true reason for worsening the Fourier coefficients $a_{i}, b_{i}$ estimation by using the rectangular $R_{c}\left(i, t_{k}\right), R_{s}\left(i, t_{k}\right)$ functions. It is shown that these functions under certain conditions actually can be used as well as the respective sinusoidal functions. To reveal what exactly these conditions are, specific properties of the rectangular functions must be taken into account. Consideration of them follows.

\subsection{Specifics of the rectangular basis functions}

The problem is that the rectangular basis functions of the considered type are not orthogonal, they have many components. Specifically, these functions contain components at odd frequencies $\omega_{i} t, 3 \omega_{i} t, 5 \omega_{i} t, 7 \omega_{i} t, 9 \omega_{i} t, 11 \omega_{i} t, \ldots$ and the amplitudes of these components at the respective frequencies are equal to $1,1 / 3,1 / 5,1 / 7$, $1 / 9,1 / 11, \ldots$. It means that whenever the input signal has components at frequencies $3 f_{i}$, $5 f_{i}, 7 f_{i}, 9 f_{i}, 11 f_{i}, \ldots$, errors will affect the values of the Fourier coefficients estimated at frequency $f_{i}$. Therefore estimation of the coefficients $a_{i}, b_{i}$, performed on the basis of functions $R_{c}\left(i, t_{k}\right), R_{s}\left(i, t_{k}\right)$, has to be done in a restricted frequency range, excluding from the input signal $x(t)$ the frequencies within the range [DC, $\left.1 / 3\left(f_{s} / 2\right)\right]$ from the full frequency range $\left(\mathrm{DC}, f_{\mathrm{s}} / 2\right)$.

What happens if this requirement is not satisfied is shown in Fig.2. Spectrum analysis of a signal, containing 6 components in the frequency range $(0,300)$, is performed in the classical way and by using the rectangular basis functions. As can be seen, the first component of the signal within the frequency range [DC, $\left.1 / 3\left(f_{s} / 2\right)\right]$, shown in the spectrogram obtained by using the classical approach to DFT, is significantly distorted in the spectrogram calculated at using the rectangular basis functions.

The necessity of such narrowing the input signal frequency range by using bandlimited filtering represents a significant disadvantage of the approach to complexityreduced DFT based on using this type of rectangular functions. In result of studies related to this problem (Sudars et al., 2016; Bilinskis et al., 2016) an approach to resolution of it has been developed. Description of it, used for avoiding this particular disadvantage of using the rectangular basis functions, follows. 

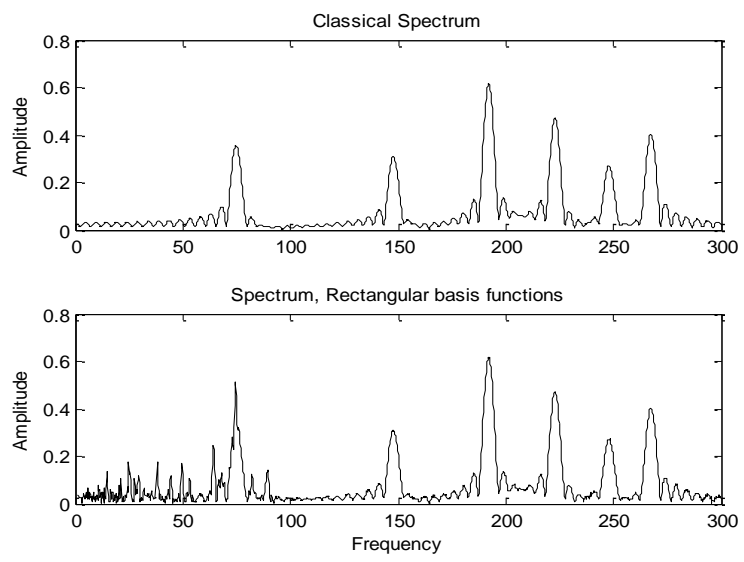

Fig.2. Two types of spectrograms obtained in the case where the signal has a component in the low frequency area.

\section{Using input signal heterodyning}

The mentioned and described above disadvantage of using rectangular basis functions for DFT can be avoided by using heterodyning of the input signals for shifting the frequencies of signal $x(t)$ to a higher intermediate frequency (IF) range. Then it is possible to use the rectangular functions $R_{c}(i, t), R_{s}(i, t)$ at performing the complexityreduced DFT of this frequency-shifted signal without distortions related to the impact of the higher frequency components at $3 \omega_{i} t, 5 \omega_{i} t, 7 \omega_{i} t, 9 \omega_{i} t, 11 \omega_{i} t, \ldots$. Diagrams given in Fig. 3 explain how that can be achieved.

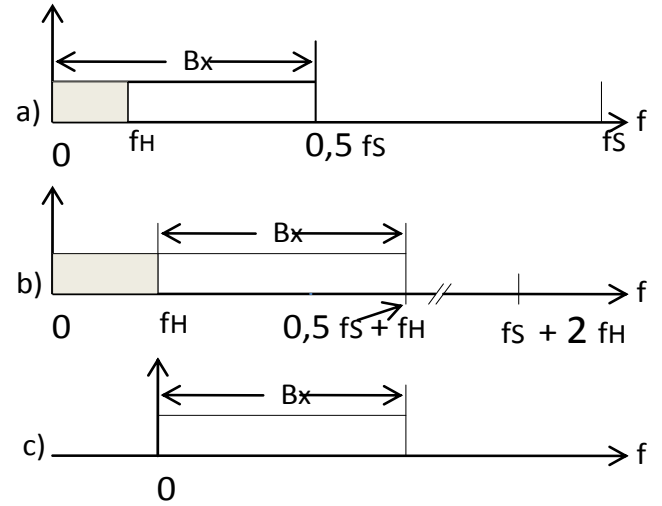

Fig.3. Diagrams illustrating frequency shifting of the input signal performed by using IF and the signal frequency multiplications (heterodyning): (a) usable frequency band of the input signal bandwidth $B_{x}$ spectrum; (b) frequency shift for $f_{H}$ leads to widening the usable frequency band so that it covers the whole bandwidth of the input signal, as it is shown in (c).

\subsection{Essence of the method}

Specifically, the diagrams in Fig.3 illustrate the fact that only the frequencies from $1 / 3\left(f_{s} / 2\right)$ to $f_{s} / 2$ of the respective signal bandwidth $B_{x}$ can be recovered from its sampled 
version by using a band-limited filter in the case where the rectangular functions $R_{c}(i, t), R_{s}(i, t)$ are used for estimating the Fourier coefficients.

The price that should be paid for using heterodyning is increasing the signal sampling frequency to $\left(f_{s}+2 f_{H}\right)$ in accordance to the diagram in Fig. $3 \mathrm{~b}$. Note that the signal $x(t)$ is then recovered in the IF range. Fortunately that does not represent a problem. The diagram in Fig.3c illustrates the simplicity of recovering the signal true frequencies. For that, the frequency scale simply should be moved to the right for the frequency interval equal to $f_{H}$.

\subsection{Simulation of using Heterodyning}

Applicability of this approach to avoiding the limitation of the input signal frequency band, imposed by using the wide-band rectangular basis functions, was investigated by computer simulations and the obtained results were positive.
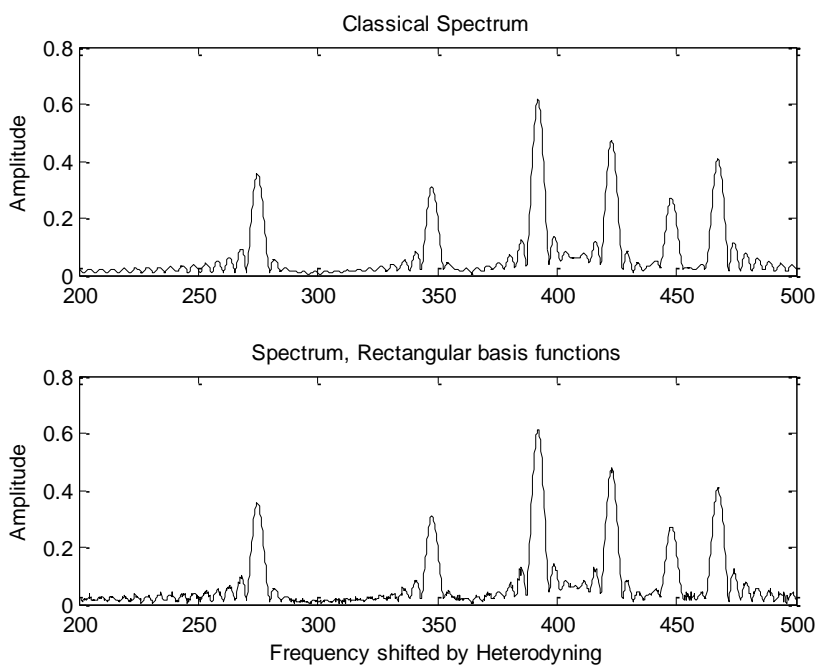

Fig.4. Illustration of the signal spectrogram in the full frequency range $\left(D C, 0.5 f_{s}\right)$ shifted in the frequency domain to the right for the interval of the heterodyning frequency $f_{H}=200$.

The spectrograms, given in Fig.4, illustrate application of heterodyning for eliminating the mentioned limitation of the input signal frequency band. The input signal contains sinusoidal components at frequencies 75, 148, 192, 223, 248, 267 and the amplitudes of these components are equal to $0.35 ; 0.3 ; 0.6 ; 0.45 ; 0.27$ and 0.4 , respectively. The classically obtained upper spectrogram shown in Fig.4 displays the input signal frequencies shifted for $f_{H}=200$. To recover the true values of these frequencies, the frequency scale of the spectrogram should be shifted to the left for the frequency interval $f_{H}=200$. It means that the spectrograms obtained by using the rectangular basis functions also shown in Fig.4 actually cover the whole frequency range of the input signal from DC up to 300 . 


\subsection{Heterodyning as part of the Analog-to-Event conversion}

Input signal $x(t)$ in this case is transformed according to the heterodyning principle. The signal frequencies are multiplied in the Mixer, shown in the structural diagram given in Fig.5, with the frequency $f_{H}$ supplied by the Oscillator. The obtained heterodyne signal then is periodically sampled in the Track and Hold mode so that the levels of the hold signal sample values can be passed to the Comparator of the Crossing Event Detector. Sampling is performed periodically at predetermined rate dictated by the output pulses of the Clock. The clock pulse frequency is quite accurate and these pulses are used also for synchronizing generation of the reference function and forming the front edges of the Pulse Width Modulated output pulses.

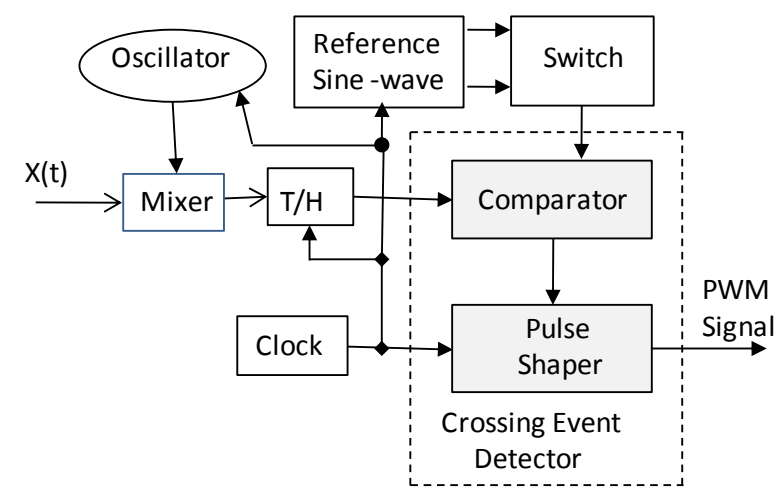

Fig.5. Block diagram of the transmitter used for transforming analog signals to PWM signals by using the considered heterodyning method.

The Track and Hold $(\mathrm{T} / \mathrm{H})$ block converts the analog input signal $x(t)$ to its stepwise approximation with the step levels equal to the respective signal sample values. The representative events in this case are formed as the crossing events of the $\mathrm{T} / \mathrm{H}$ output signal with a specific reference function. The reference function in this case is formed from the direct and inverted half-waves of the generated reference sine-wave. The Switch forms the doubled frequency reference function and Pulse Shaper forms the output pulse width modulated signals. While rise times of these pulses are formed at the sampling time instants, the pulse fall edges mark the time instants of the representative event happening. The formed PWM signal then is transmitted over the optical lines where it is demodulated and processed.

\subsection{Taking heterodyning into account}

In general, using the mentioned rectangular functions as the basis for estimating the Fourier coefficients is done according to equations (3). However definition of these functions in this case differs. It is specific as estimating the Fourier coefficients $a_{i}, b_{i}$ for the input signal component at the frequency $f_{i}$ is performed by using the frequency $\left(f_{i}+f_{H}\right)$. Evidently then

$$
\left\{\begin{array}{l}
R_{c}\left(i, t_{k}\right)=\operatorname{sgn}\left[\cos \left(2 \pi\left(f_{i}+f_{H}\right) t_{k}\right)\right] \\
R_{s}\left(i, t_{k}\right)=\operatorname{sgn}\left[\sin \left(2 \pi\left(f_{i}+f_{H}\right) t_{k}\right)\right]
\end{array}\right.
$$


Actually taking into account the effect due to application of heterodyning is simple enough and does not complicate the involved calculations. Using heterodyning represents the first principal improvement of the approach to DFT based on using the binary $(-1,+1)$ valued rectangular functions $R_{c}\left(i, t_{k}\right), R_{s}\left(i, t_{k}\right)$. Brief description of implementing the second type of improvement (suggested in (Sudars et al., 2016; Bilinskis et al., 2016)), follows.

\section{DFT of the transmitted PWM signals}

The difference between the results obtained at spectrum analysis by using either the sinusoidal or the rectangular basis functions is considered as presence of the noise degrading DFT results in the second case. At first glance it seems that these results must be worse because the basis functions $R_{c}\left(i, t_{k}\right), R_{s}\left(i, t_{k}\right)$ then significantly differ from the sine-wave basis functions used at performing DFT in the classical way. Actually interpretation of the rectangular functions as an approximation of the sine-wave functions does not lead to the right explanation of the role they play at DFT as it is shown in (Sudars et al., 2016).

To improve DFT precision by significant reduction of the noise, related to the widespread spectrum of the used functions $R_{c}\left(i, t_{k}\right), R_{s}\left(i, t_{k}\right)$, they have to be sampled at a relatively high frequency whenever the time instants of switching the rectangular functions from one binary value to other do not coincide with the signal sampling time instants. Sampling frequency used for digitizing the input signal $x(t)$, determined by taken into account the highest frequency of its components, usually is lower. In other words, requirements to the sampling rates to be used for digitizing the input signal $x(t)$ and the generated rectangular functions $R_{c}\left(i, t_{k}\right), R_{s}\left(i, t_{k}\right)$ then are different.

\subsection{Using two-rate sampling at DFT.}

Actually sampling of the rectangular basis functions must be performed at a significantly higher frequency for suppressing the noise due to aliasing of the rectangular function components. The problem is that suppressing this type of noise requires significant increasing the frequency of sampling these functions. Using a single source of the sampling frequency for digitising the input signal and the basis functions then represents a problem as taking and processing many signal sample values at a higher sampling frequency would significantly complicate digital signal processing performed at the spectrum analysis.

An approach to resolution of the mentioned problem has been found. It is based on sampling the signal and the rectangular basis functions separately at two different sampling rates. Then the used rectangular basis functions are sampled with a small time step providing for precise timing the events when the values $(-1,+1)$ of these functions change the polarity. This approach has been computer simulated with the focus on the achievable suppression rate (SR) of the difference between the spectra obtained by using the sine-wave and rectangular basis functions. Brief description of the obtained results follows.

The spectrum, given in Fig.6, is obtained at using 2 times higher frequency $f_{s R}$ for sampling the rectangular basis functions than frequency $f_{s}$ used for sampling the input signal. The noise overlapping the spectrum, due to the mentioned aliasing effect, in this case is noticeable. Increasing the frequency of sampling the basis function only two 
times (in comparison with $f_{s}$ ) clearly is not enough. The signal $x(t)$ in this case has components at frequencies 115, 135, 185, 210, 237 and the amplitudes of these components are equal to $0.5,0.2,0.6,0.3$ and 0.55 , respectively. This signal is sampled at frequency $f_{s}=512$ and the processed number of the signal sample values is $N=1024$.
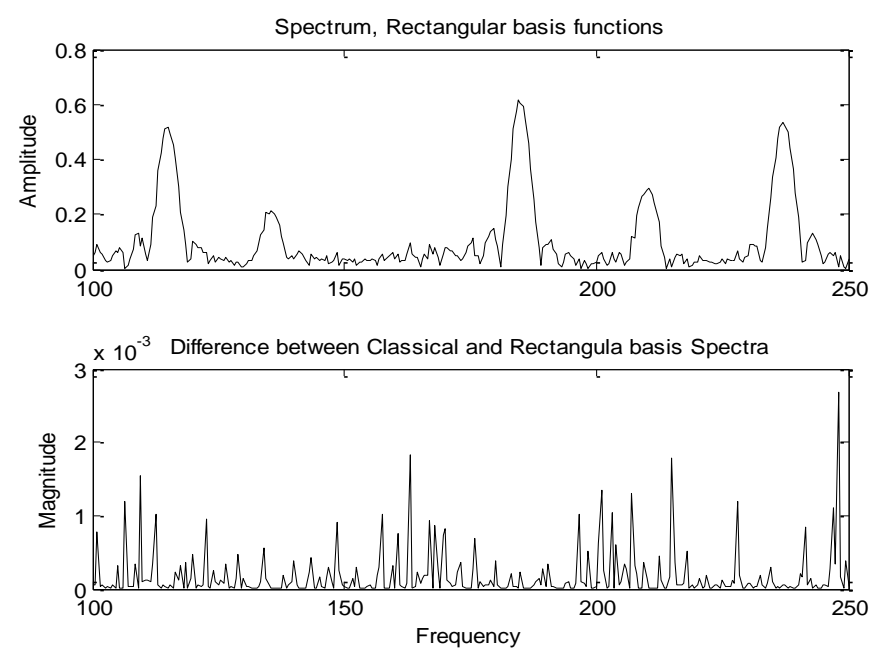

Fig.6. Spectrum (upper diagram) of the signal $x(t)$, obtained at using the rectangular basis functions sampled at two times higher frequency $f_{s R}$ than the frequency $f_{s}$ used for sampling $x(t)$.

The squared difference between the spectra obtained in the classical way and by using the rectangular basis functions (lower diagram).

Much better are the results obtained in result of using eight times higher frequency for sampling the rectangular basis functions. The improvement achieved under this condition is illustrated in Fig.7.
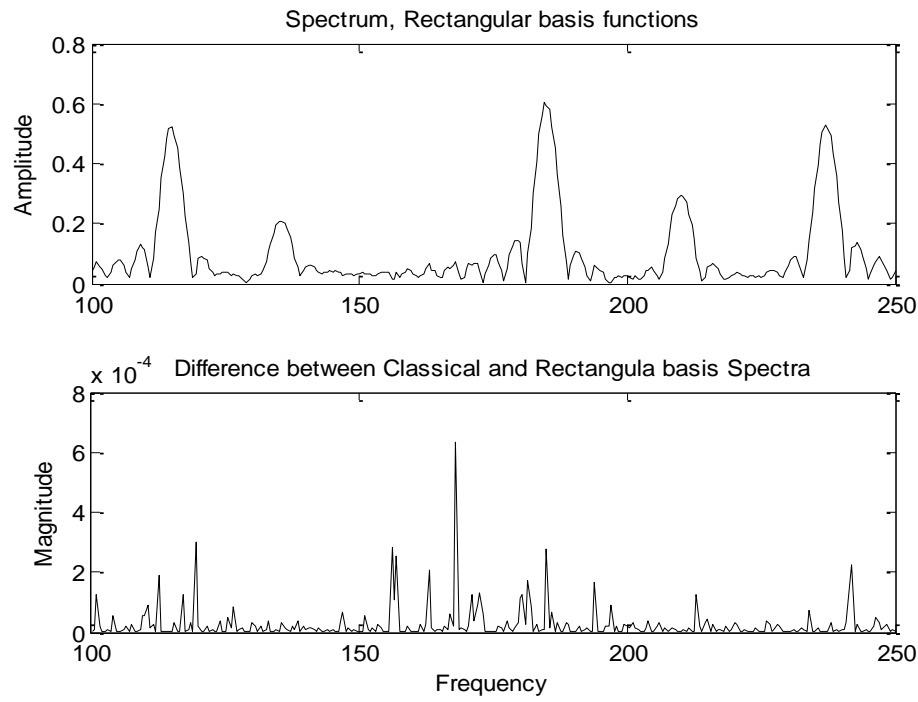

Fig.7. Spectrum obtained at sampling the rectangular basis functions in the case where $f_{s R}=8 f_{s}$ (upper diagram); the squared difference between the spectra estimated by using the sinusoidal and rectangular basis functions (lower diagram). 
As it is shown in Fig.7, the difference between both spectra obtained in the considered ways is significantly smaller in the case where 8 times higher sampling frequency $f_{s R}$ is used.

\subsection{Improvement achievable by increasing the sampling frequency $f_{s R}$}

The rate of suppressing (SR) the mean power of the difference between the spectra estimated by using the sinusoidal and rectangular basis functions evidently depends on the parameters of the respective signal and on the ratio $f_{s R} / f_{s}$ of the sampling frequencies. The numerical values given in Table 1 show how this rate SR and the average sample value $\mathrm{AD}$ of the mentioned difference between the estimated spectra is changing in response to the higher ratio $f_{s R} / f_{s}$ values in the specific case where the input signal has the components with the parameters given above.

Table 1
\begin{tabular}{|c|c|c|c|c|}
\hline$f_{s R} / f_{s}$ & 2 & 4 & 8 & 16 \\
\hline $\mathrm{SR}$ & 1 & 2.99 & 6.89 & 12.11 \\
\hline $\mathrm{AD}$ & 0.0129 & 0.0074 & 0.0049 & 0.0037 \\
\hline
\end{tabular}

The plot in Fig.8 illustrates how the precision of performing DFT of the considered signal is improving at using increased frequencies for sampling the rectangular basis functions at estimation of the Fourier coefficients. It is built by using the numerical data given in Table 1.

The difference between the spectra estimated by using the sinusoidal and rectangular basis functions is considered as the noise introduced by using the rectangular functions. Actually power of this noise is not so high even at moderate increasing the frequency of sampling this type of the basis functions. As it can be seen in Fig.8, while increasing the sampling rate $f_{s R} / f_{s}$ for $2,4,8$ times leads to significant reducing this noise, further increasing the sampling frequency $f_{s R}$ up to the 16 times higher level than $f_{s}$ provides for gaining relatively much smaller decreasing of the noise. It seems that sampling the rectangular basis functions at the rate 8 times higher than the rate of sampling the input signal should be acceptable in a wide application area.

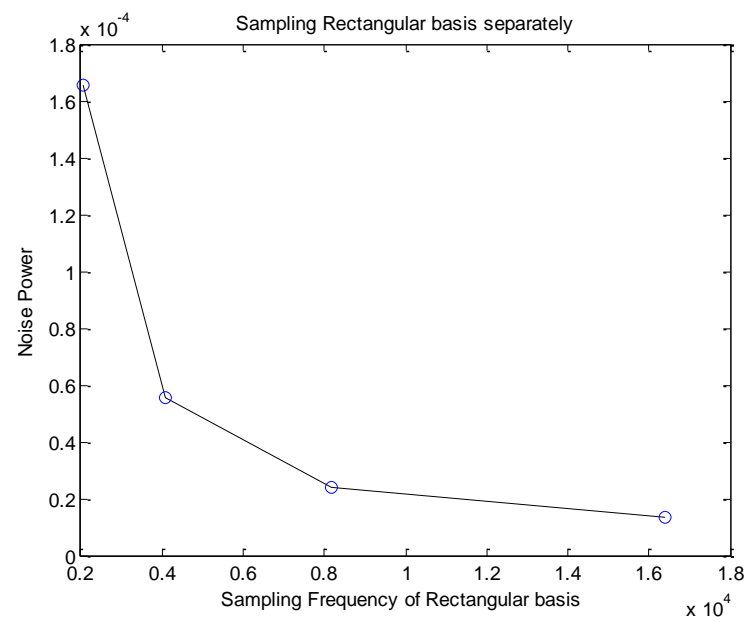

Fig.8. Typical achievable rate of suppressing the mean power of the difference between the spectra estimated by using the sinusoidal and rectangular basis functions. 


\section{Conclusions}

Specifics of using high performance event timers for signal transmission and representation in the time and frequency domains are considered and described.

Representing the transmitted signal in the Time domain is performed by the input signal sample value recovering from the transmitted width modulated pulse stream. It is essential to use the picosecond resolution high-speed Event Timer A033-ET systems for recovery the transmitted signal sample values as 12 bit numbers from each transmitted single pulse carrying a 12 bit word.

More complicated is representing the transmitted signal in the frequency domain. That is done on the basis of Fourier coefficient estimation and the used approach is based on replacing the sinusoidal basis functions by binary $(+1,-1)$ valued rectangular functions. Assumption that these functions represent a rough approximation of the sinusoidal functions actually is wrong. It is shown that under certain conditions the rectangular basis functions can be used as well as the respective sinusoidal functions. These conditions are revealed and discussed.

Firstly, they are well applicable if the input signal frequency band does not cover the frequencies from 0 up to $1 / 3$ of the Nyquist frequency. The second condition is that the time instants of switching the rectangular functions from one to other binary values must coincide with the signal sampling time instants.

The following two methods are suggested which can be used in the cases where these two requirements are not met:

- $\quad$ Shifting the input signal frequencies by heterodyning;

- Using different sampling rates for digitizing the input signals and the rectangular basis functions.

In particular it is shown that sampling the rectangular basis functions at the rate 8 times higher than the rate of sampling the input signal should be acceptable in a wide application area.

\section{References}

Artyukh, Yu., V. Bespal'ko, E. Boole, and V. Vedin (2011). „Event Timer A033-ET: Current State and Typical Performance Characteristics". In: Proc. 17th International Workshop on Laser Ranging, Bad Kotzting, Germany, May 16-20, 2011, pp. 107-110.

Bilinskis, I. (2007). Digital Alias-free Signal Processing, John Wiley \& Sons, Ltd (UK), 430 p.

Bilinskis, I. and K. Sudars (2008a). „Digital representation of analog signals by timed sequences of events". In: Electronics and Electrical Engineering, No. 3(83), March, 2008.

Bilinskis, I. and K. Sudars (2008b). „Specifics of constant envelope digital signals”. In: Electronics and Electrical Engineering, No. 4(84), March, 2008.

Bilinskis, I., E. Boole, K. Sudars, and V. Vedin (2013a). „Digital Representing of Analog Signals Using Event Timing Information". In: Automatic Control and Computer Sciences, 2013, Vol. 47, No. 6, pp.300-309.

Bilinskis, I., E. Boole, A. Mezerins, and V. Vedin (2013b). „Alias-free compressed signal digitizing and recording on the basis of Event Timer". In: Proc. of 21st Telecommunications Forum TELFOR, Serbia, Belgrade, November 26-28, 2013, pp. 443-446.

Bilinskis, I., E. Boole, and A. Skageris (2015). "Data acquisition and transmission based on application of high performance Event Timers". In: Proc. 1-st International IEEE Conference on Advances in Wireless and Optical Communications (RTUWO-2015), 2015. 
Bilinskis, I., E.Boole, and A.Skageris (2016). " Discrete Fourier Transform of event timing signals transmitted over optical lines". In: Proc. 2-nd International IEEE Conference on Advances in Wireless and Optical Communications (RTWO-2016) , Riga, 2016, pp.33-36.

Curtis, S.R. and A.V. Oppenheim (1987). „Reconstruction of multidimensional signals from zero crossings". In: J. Opt.Soc. Am. A, vol.4, pp.221-231, January 1987.

Kumaresan, R. and Y. Wang (2001). „On representing signals using only timing information”. In: Journal of Acoustic Society of America, 110 (5), Pt. 1, Nov. 2001.

Sudars, K., I. Bilinskis,E. Boole, and V. Vedin (2015). "Signal Analog-to-Event-to-Digital converting based on periodic sampling and precise event timing". In: Proc. 25th International Conference Radioelektronika 2015 (MAREW-2015), 2015, pp.133-136.

Sudars, K., I.Bilinskis, and E.Buls (2016). "Discrete Fourier Transform of the signals recovered by using high-performance Event Timers". In: Proc. 15th Biennial Baltic Electronics Conference (BEC-2016), Tallinn, 2016, pp.139-142.

Zhongping, Z., Z. Haifeng, Y. Fumin, W. Zhibo, C. Juping, and Yu. Artyukh (2008). "Applications of Riga Event Timer at Shanghai SLR Station". In: Proc. 16th International Workshop on Laser Ranging, Poland, Poznan, October 13-17, 2008, pp.447-453.

Received November 28, 2016, accepted February 4, 2017 